\title{
An analysis of near-circular lunar mapping orbits
}

\author{
R V RAMANAN* and V AdimuRthy \\ Aeronautics Entity, Vikram Sarabhai Space Centre, Thiruvananthapuram 695 022, India. \\ *e-mail: rv_ramanan@vssc.org
}

\begin{abstract}
Numerical investigations have been carried out to analyse the evolution of lunar circular orbits and the influence of the higher order harmonics of the lunar gravity field. The aim is to select the appropriate near-circular orbit characteristics, which extend orbit life through passive orbit maintenance. The spherical harmonic terms that make major contributions to the orbital behaviour are identified through many case studies. It is found that for low circular orbits, the 7 th and the 9th zonal harmonics have predominant effect in the case of orbits for which the evolution is stable and the life is longer, and also in the case of orbits for which the evolution is unstable and a crash takes place in a short duration. By analysing the contribution of the harmonic terms to the orbit behaviour, the appropriate near-circular orbit characteristics are identified.
\end{abstract}

\section{Introduction}

The behaviour of satellites orbiting the Moon is mainly influenced by the non-spherical gravity field of the Moon. Many lunar missions demand a long operational life to conduct scientific experiments. For example, the Indian Moon mission Chandrayaan-1 is conceived to have an operational life of two years in a circular polar orbit. To achieve this operational life, regular orbit maintenance to keep the spacecraft in the envisaged orbit is required. These manoeuvers require a large amount of fuel. The limitations on the availability of fuel in the spacecraft for this purpose, restricts the operational life of the moon orbiting satellites. It is desirable to use the lunar gravity field to design orbits that require minimum fuel for maintenance. Study (Cook 1991) indicates that by appropriate choice of orbit characteristics the natural variations in the orbits can be reduced. Frozen orbits, for which there is no long-term change in eccentricity and argument of periapsis, were suggested in this study. To obtain a frozen orbit one chooses an eccentricity and an argument of periapsis for a given semi-major axis and inclination.

Cook (1991) developed closed form expressions to find the eccentricity for a given inclination and this combination provides stability in the orbit evolution. These closed form solutions are the functions of the lunar gravity field which consider only zonal terms. He identified three inclinations, which provide frozen orbits for $150 \mathrm{~km}$ circular orbits. Park and Junkins (1995) used the same closed form expressions and arrived at frozen orbit parameters with updated gravity models. They identified an inclination of $101.5^{\circ}$ as the candidate for $100 \mathrm{~km}$ circular orbits. The limitations of the frozen orbit concept for circular orbits are discussed in literature (Cook and Sweetser 1992) and strategies for orbit maintenance are proposed. With tighter mission specifications on eccentricity and argument of periapsis frequent manoeuvers are envisaged. Also, frozen orbit parameters are obtained using the same formulation with the zonal terms of the LUN60D gravity model and it is concluded therein that the determination of a polar circular frozen orbit is inconclusive (Konopoliv et al 1993). A severe variation in the life times predicted with different gravity fields, is also reported in the literature.

Accurate gravity model is required especially with low altitude orbits. Gravity models for the Earth with zonal terms only, yield reasonably good approximations to the actual field in orbit

Keywords. Lunar mapping; orbit maintenance; orbit life time; lunar gravity model; spherical harmonics. 
Table 1. Gravity models and orbital life time estimates.

\begin{tabular}{lc}
\hline \multicolumn{1}{c}{ Model } & $\begin{array}{c}\text { Life time for } 100 \mathrm{~km} \\
\text { circular }\end{array}$ \\
\hline LUN60D $(60 \times 60)$ & 193 \\
GLGM2 $(70 \times 70)$ & $>2$ years \\
LP100J $(100 \times 100)$ & 160 \\
\hline
\end{tabular}

Table 2. Harmonic coefficients and the orbital life time.

\begin{tabular}{cc}
\hline $\begin{array}{c}\text { LP100J } \\
\text { degree } \times \text { order }\end{array}$ & $\begin{array}{c}\text { Life time for } 100 \mathrm{~km} \\
\text { circular polar } \\
\text { orbit (days) }\end{array}$ \\
\hline $100 \times 100$ & 160.400 \\
$70 \times 70$ & 161.569 \\
$50 \times 50$ & 160.611 \\
$40 \times 40$ & 155.785 \\
$30 \times 30$ & 130.646 \\
$20 \times 20$ & 222.410 \\
\hline
\end{tabular}

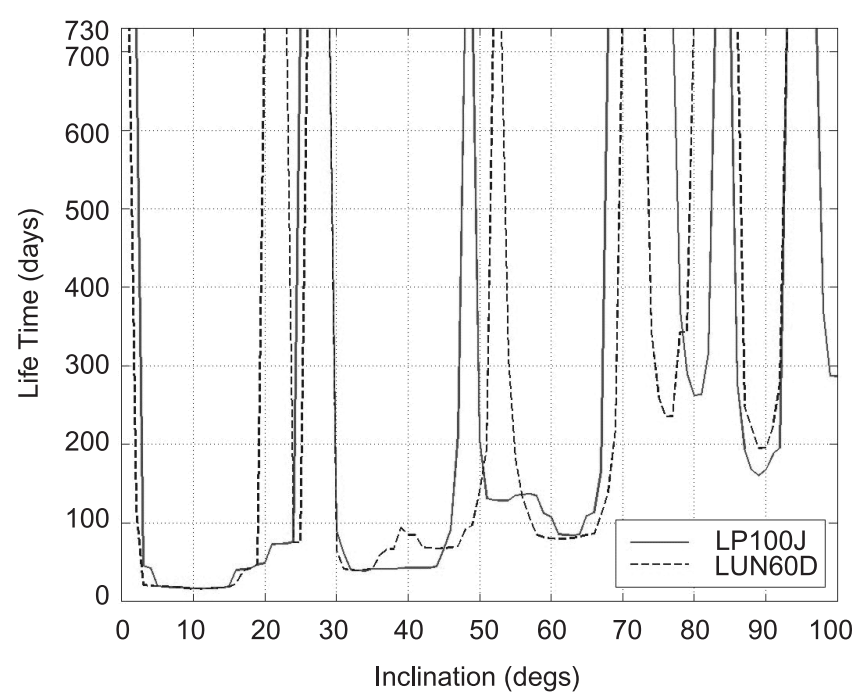

Figure 1. Impact of inclination on the orbital life time.

evolution studies. However, with the moon's gravity field heavily dependent on longitudinal variations, it becomes necessary to include non-zonal terms also in the orbital evolution process. In this paper, we identify a gravitationally stable lunar mapping orbit which is circular and near-polar by including the full gravity model. Because of the limitations of the closed form solutions for circular orbits and the necessity of non-zonal terms for accurate orbit evolution, investigations are carried out using Cowell's integration procedures. The life times of the spacecraft are assessed for various orbit characteristics, and a stable orbit characteristic is identified for circular near-polar orbit. Further, the spherical harmonic terms (zonal as well as tesseral) that make major contributions to the orbital evolutions, are identified.

\section{Simulation model}

The simulations are carried out using the equations of motion representing the inverse square field together with the non-spherical lunar gravity field. The lunar gravity potential, $U$, is modeled in spherical harmonics using the expression,

$$
\begin{aligned}
U= & \frac{G M}{r} \sum_{l=0}^{\infty}\left(\frac{a_{e}}{r}\right)^{l} \\
& \times \sum_{m=0}^{l}\left\{\bar{C}_{l m} \cos m \lambda+\bar{S}_{l m} \sin m \lambda\right\} \bar{P}_{l m}(\sin \phi),
\end{aligned}
$$

where $r$ is the selenocentric radius, $\phi$ and $\lambda$ are the selenocentric latitude and longitude respectively; $\bar{C}_{l m}$ and $\bar{S}_{l m}$ are the normalized potential coefficients; $\bar{P}_{l m}$ are the normalized associated Legendre functions of degree $l$ and order $m ; a_{e}$ is the lunar equatorial radius; $G$ is the universal constant of gravitation, and $M$ is the lunar mass. Gravity models provide the potential coefficients. Other perturbing forces from the Earth and the Sun are not included, because they do not cause much variation in life time assessment studies.

\section{Lunar gravity models}

The gravity field for the Moon has been determined by the measurements carried out by several missions. Though many gravity field models do exist in the literature, three gravity models represent the ongoing evolutionary process. The first elaborate and complete model LUN60D (Konopoliv et al 1993), a $60 \times 60$ gravity model, was determined by earlier lunar orbiter and Apollo missions. However, many of these missions were with low inclination lunar parking orbits and the measurements covered only the equatorial region of the near side (visible to the Earth). With more data from the highly inclined orbit, Clementine mission provided GLGM2 (Lemoine et al 1997), a $70 \times 70$ gravity model. Lunar Prospector Mission was in polar orbit and covered the earlier uncovered regions of near side, and based on these data gravity models of degree and order of up to 160 have been developed (Konopoliv et al 1998). LP100J is one such $100 \times 100$ model.

In an exercise to determine the choice of gravity models for this study, life times of the moon orbiting satellites are assessed with the three gravity models. Table 1 presents the orbital life times of a $100 \mathrm{~km}$ circular polar orbit with these gravity models. The huge differences indicate the inadequacy of the models. Of these a $100 \times 100$ model LP100J and LUN60D are considered for a study in which the predicted life times are close to the 


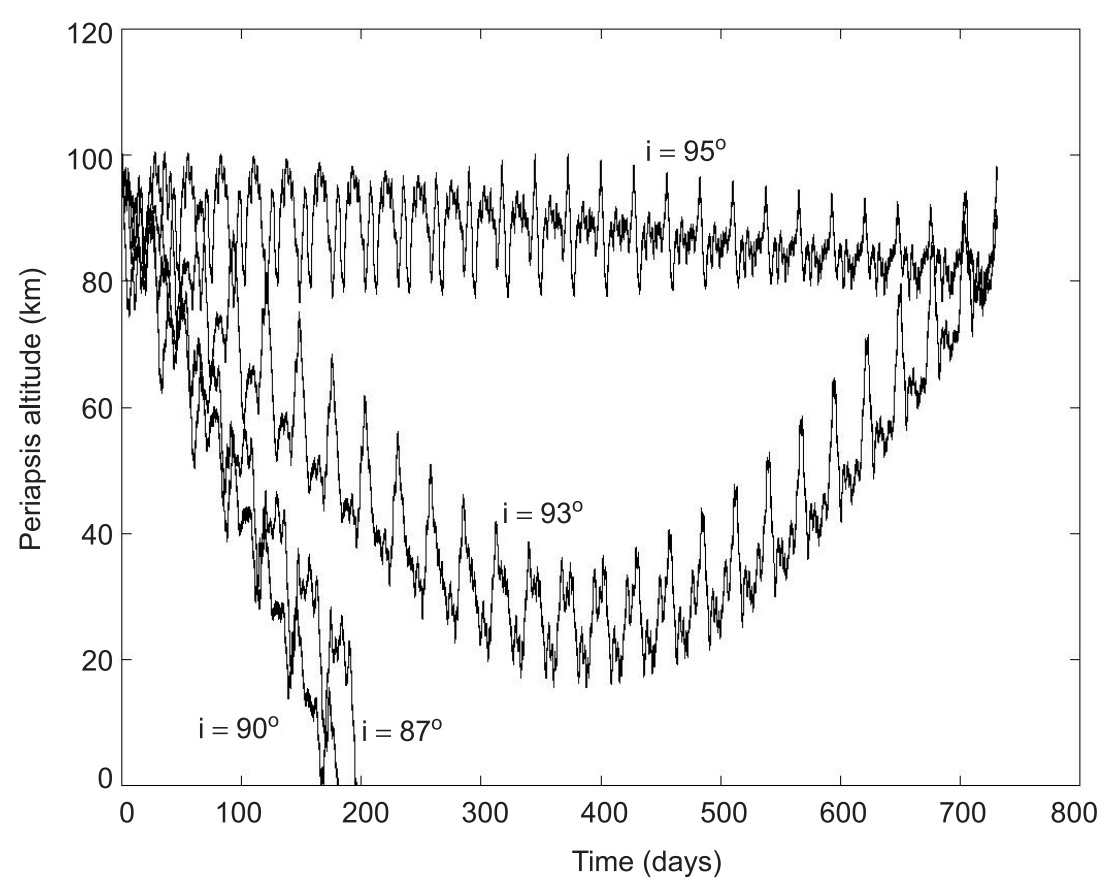

Figure 2. Evolution of periapsis altitude for different inclinations.

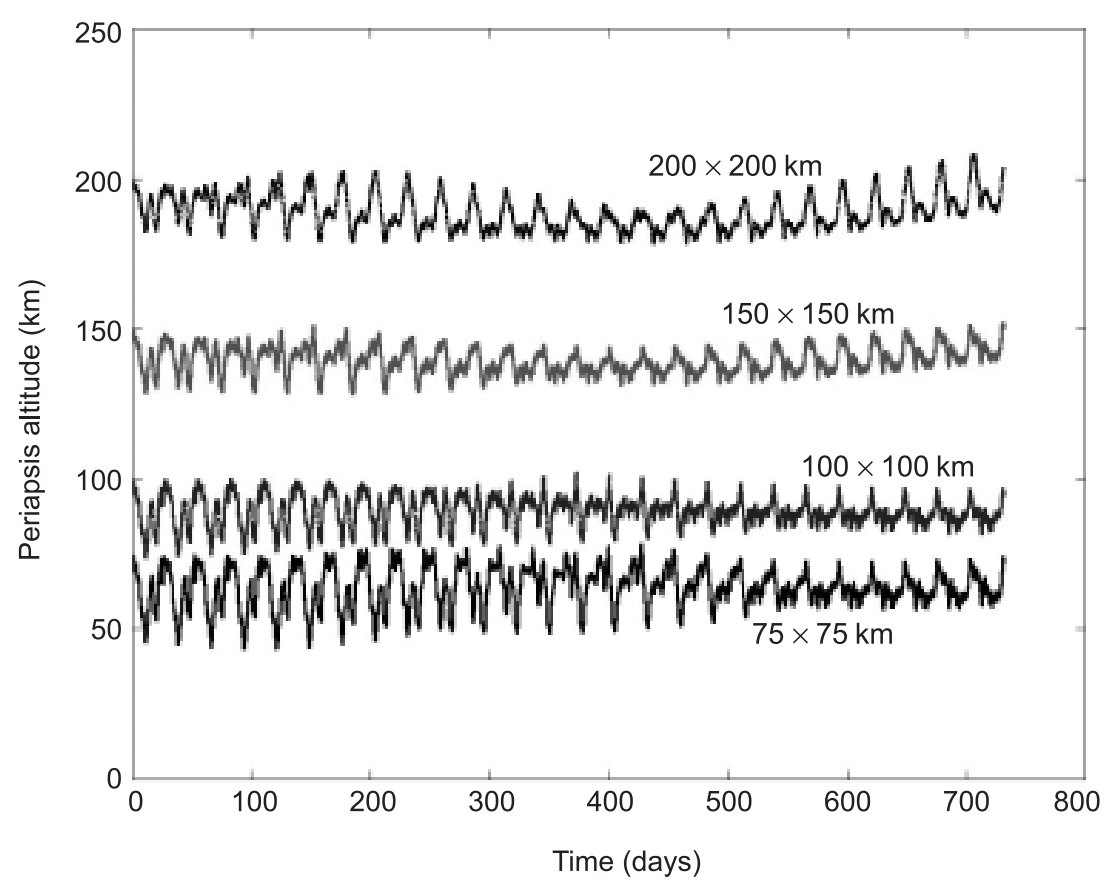

Figure 3. Evolution of periapsis altitude for different orbit sizes.

ones reported in the literature (Lozier et al 1998). The investigations being numerical, it is natural to attempt to reduce the gravity model coefficients without compromising the accuracies to save computational time. LP100J, a $100 \times 100$ model, is considered for this exercise. Table 2 predicts the orbital life time for a $100 \mathrm{~km}$ circular polar orbit, for various models that are truncated LP100J models. Other initial orbital characteristics (argument of periapsis, right ascension of ascending node and mean anomaly) are set to zero. A $50 \times 50$ model predicts the life time with less than $0.5 \%$ error, and hence considered for further investigations. Prediction accuracy suffers severely with any further reduction of the model. Though, the analysis for choice of truncated model is provided for one orbit, similar behaviour is expected for other orbits. 

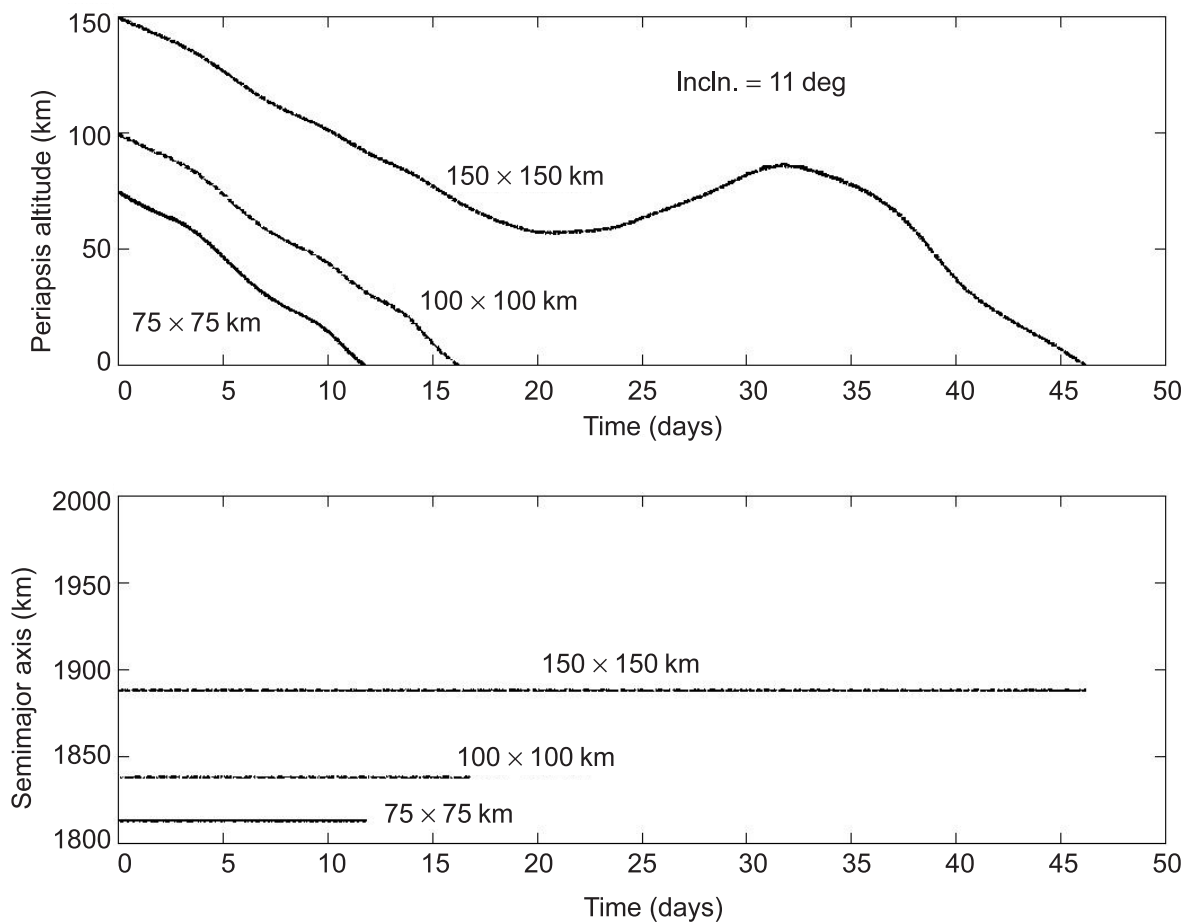

Figure 4. Evolution of periapsis altitude and semi-major axis.

Table 3. Impact of right ascension of ascending node on orbit evolution.

\begin{tabular}{rccc}
\hline & & \multicolumn{2}{c}{ Inclination $=95^{\circ}$} \\
\cline { 3 - 4 } RAAN $\left(^{\circ}\right)$ & $\begin{array}{r}\text { Inclination }=90^{\circ} \\
\text { Life time }(\text { days })\end{array}$ & Life time $($ days $)$ & $\begin{array}{c}\text { Minimum periapsis } \\
\text { altitude }(\mathrm{km})\end{array}$ \\
\hline 0.0 & 167.45 & $>2$ years & 74.0 \\
30.0 & 167.42 & $>2$ years & 52.78 \\
60.0 & 172.01 & $>2$ years & 57.42 \\
90.0 & 174.94 & $>2$ years & 50.31 \\
120.0 & 203.25 & $>2$ years & 57.30 \\
150.0 & 179.32 & $>2$ years & 60.69 \\
180.0 & 175.92 & $>2$ years & 54.18 \\
210.0 & 181.35 & $>2$ years & 53.20 \\
240.0 & 183.65 & $>2$ years & 56.80 \\
270.0 & 180.45 & $>2$ years & 57.20 \\
300.0 & 180.74 & $>2$ years & 69.42 \\
330.0 & 165.64 & & 70.53 \\
\hline
\end{tabular}

\section{Behaviour of near-circular lunar mapping orbits}

The spacecraft in a $100 \mathrm{~km}$ circular polar orbit has an orbital life time of about 160 days only due to the gravity field. Orbit maintenance manoeuvers are required to keep periapsis within the mission specifications $( \pm 20 \mathrm{~km}$ in the case of Lunar Prospector (Lozier et al 1998). As pointed out earlier, appropriate initial orbit characteristics can extend the life and reduce the maintenance cost. With the orbit size and shape fixed as circular, the parameters which can be changed are inclination and the right ascension of ascending node. The contribution of these parameters to the orbital behaviour in the presence of the gravity field is studied. Appropriate values for these parameters are selected. The contribution of spherical harmonics of the lunar gravity field to the orbital evolution is analysed.

\subsection{Impact of inclination}

The orbital life times of a $100 \mathrm{~km}$ circular orbit has been assessed for different inclinations, and are plotted in figure 1. The right ascension of 

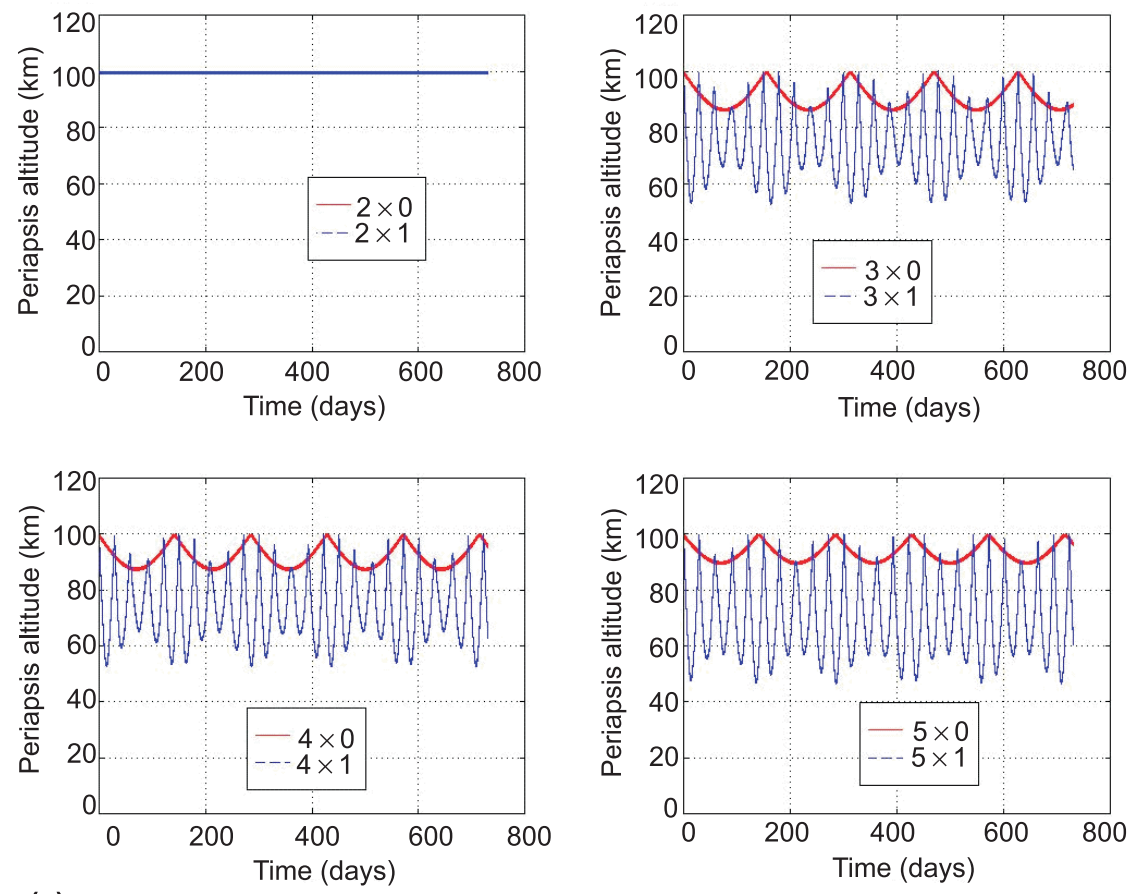

(a)
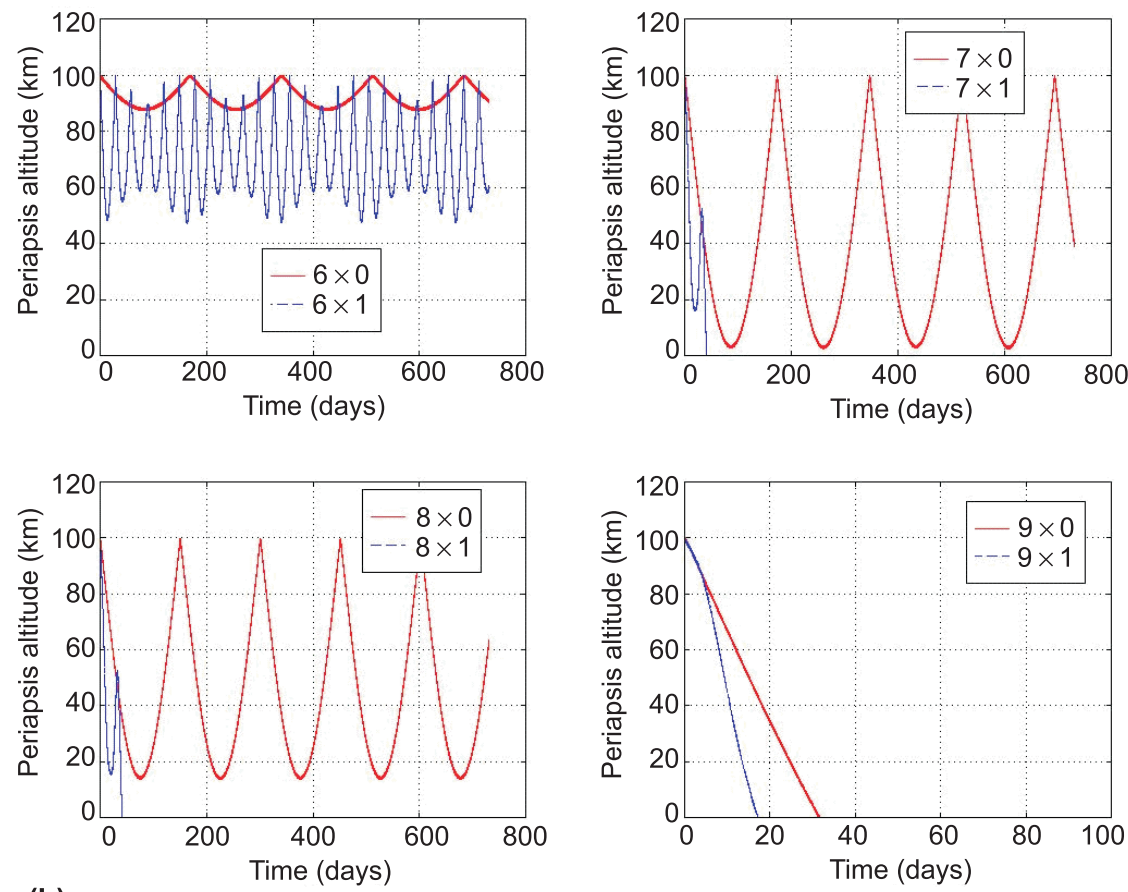

(b)

Figure 5. Contribution of spherical harmonics to the orbit evolution $\left(i=11^{\circ}\right)$.

ascending node is set to zero for this figure. The strong dependence of the orbital life time on orbital inclination is clearly seen. The life time estimates vary from a minimum of 16 days to more than 2 years duration for the same size of the orbit. The trends are the same for both the gravity models under consideration. It is interesting to note that there exist a few regions of inclinations that yield long life times. The minimum orbital life time occurs when the inclination is about $11^{\circ}$. The orbital periapsis altitude behaviour for some inclinations around $90^{\circ}$ is plotted in figure 2 . The stability of the orbit for $i=95^{\circ}$ can be noticed while for $i=90^{\circ}$, the orbit decays rapidly. The maximum absolute variation is about $26 \mathrm{~km}$ only in two years for $i=95^{\circ}$. This orbit inclination keeps the 

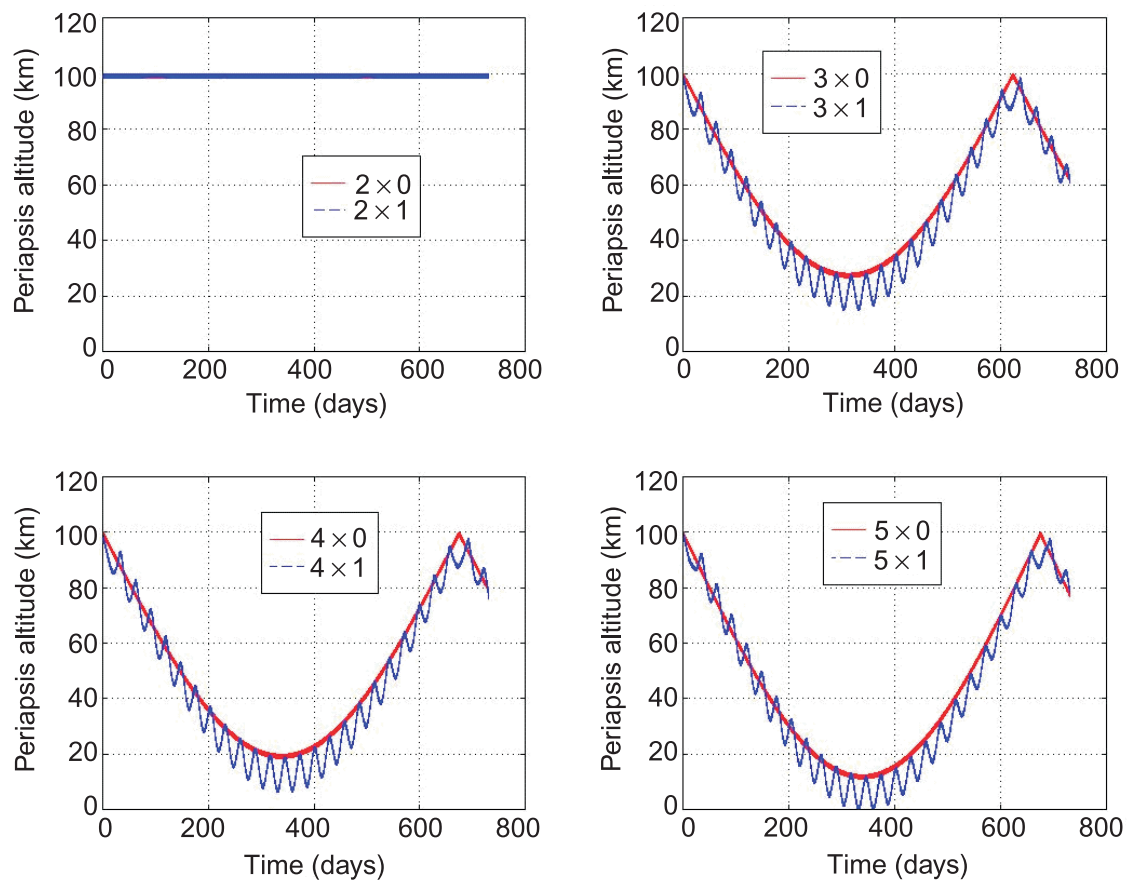

(a)
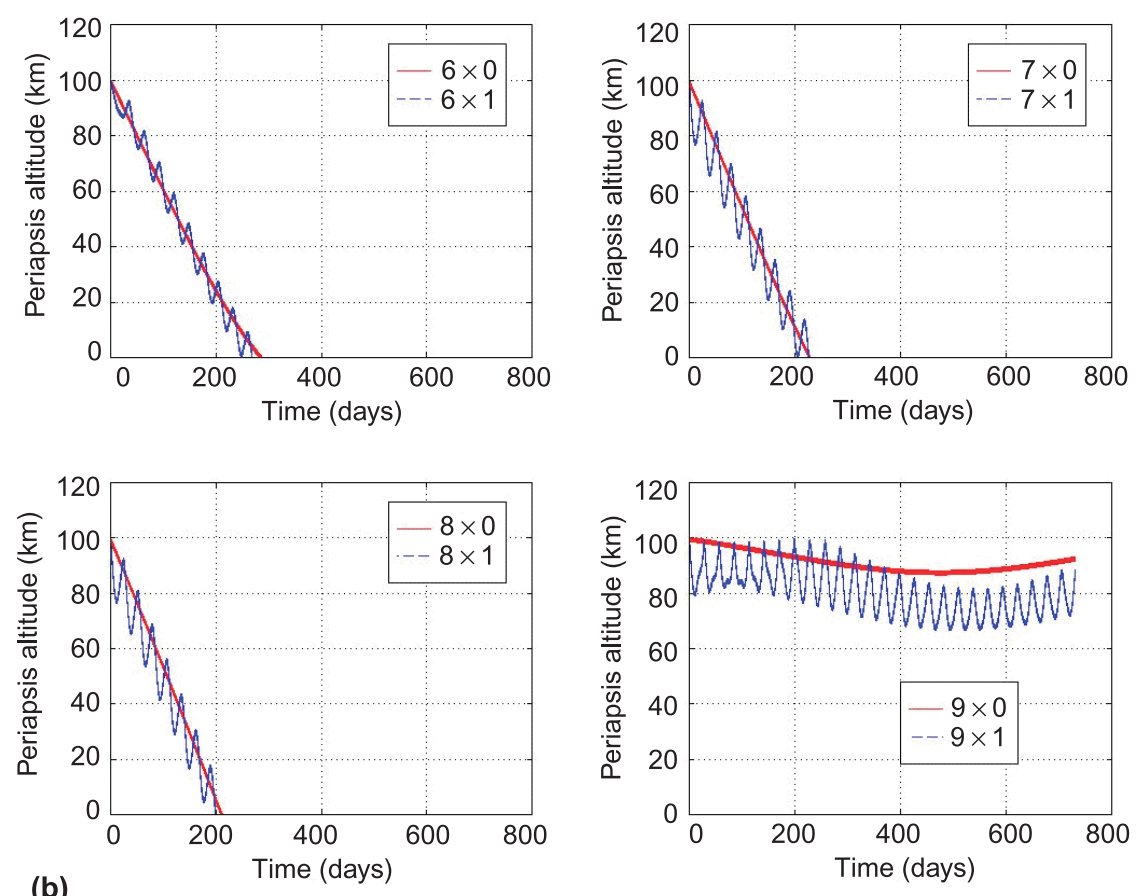

Figure 6. Contribution of spherical harmonics to the orbit evolution $\left(i=95^{\circ}\right)$.

variation of periapsis altitude at a very low level for other circular orbits of 75, 150 and $200 \mathrm{~km}$ also. This is illustrated in figure 3 .

The evolution of the periapsis altitude and the semi-major axis for $i=11^{\circ}$, the minimum life time case, is given in figure 4 . Though the semi-major axis remains constant in all the cases, the severe eccentricity variation due to the lunar gravity accelerates the impact of the spacecraft. For high as well as for low circular orbits, a similar trend is noticed.

\subsection{Impact of right ascension of ascending node (RAAN)}

Proper choice of this parameter could further extend the life and reduce the maintenance cost. This parameter is measured in the IAU (International 


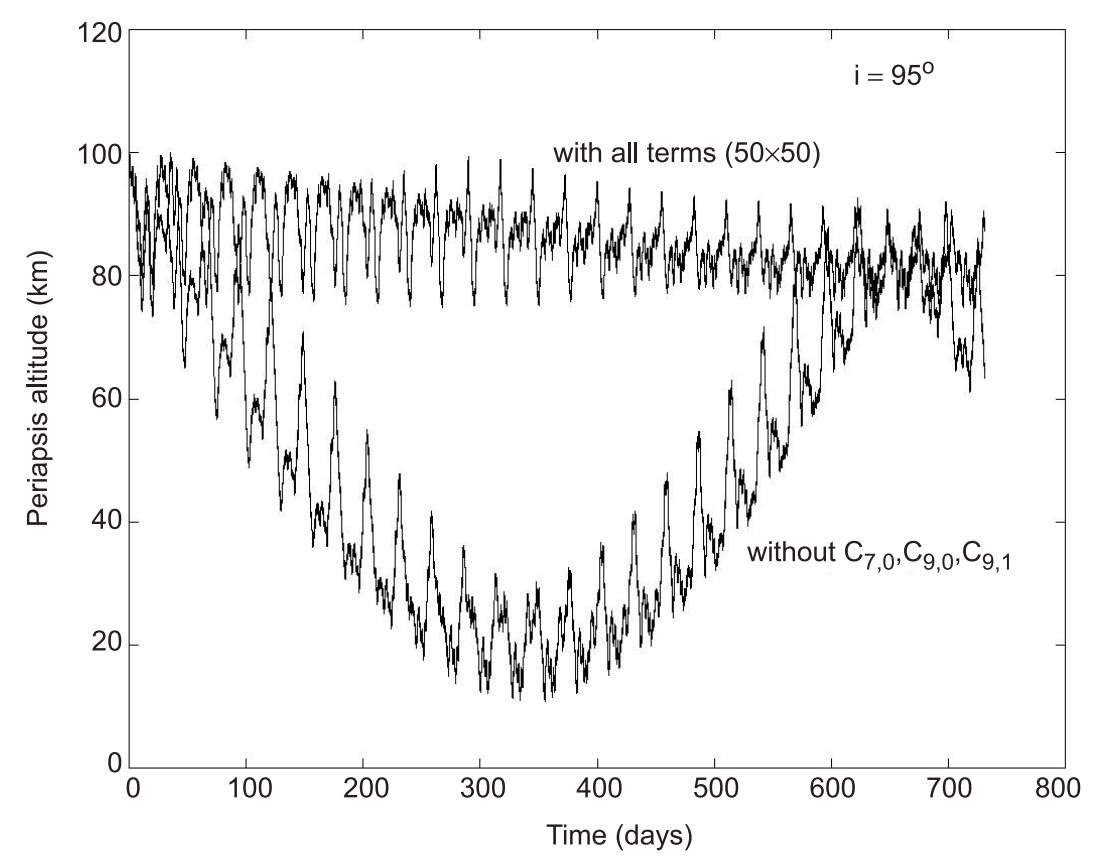

Figure 7. Impact of the spherical harmonics $\mathrm{C}_{7,0}, \mathrm{C}_{9,0}, \mathrm{C}_{9,1}$ on the periapsis altitude evolution.

Astronomical Union) coordinate frame. For inclination of $95^{\circ}$, when RAAN is in the range of (300 to $360)^{\circ}$, the minimum periapsis altitude in two years is about $70 \mathrm{~km}$ (see table 3). This kind of periapsis evolution reduces not only the cost of maintenance but also the frequency of the maintenance, and hence this range is preferable. For the polar orbits, a value of about $120^{\circ}$ for RAAN extends the life by about 35 days. Force model in these investigations include the third body effects of the Earth and the Sun. It is pointed out that RAAN does not affect the orbit evolution in the absence of non-zonal terms.

\subsection{Contribution of harmonics}

Numerical investigations are carried out to find out the contribution of the harmonics. The inclination $i=11^{\circ}$ for which rapid decay takes place and the inclination $i=95^{\circ}$ for which the long life of the craft exist, are considered for the study. These are presented in the figures 5 and 6 . Clearly, the spherical harmonic terms $\mathrm{C}_{7,0}, \mathrm{C}_{9,0}$ and $\mathrm{C}_{9,1}$ make major contributions to the orbital evolution of all the cases. When $\mathrm{C}_{7,0}$ is included in the force model the periapsis altitude variation is more than $90 \mathrm{~km}$. Inclusion of $\mathrm{C}_{9.0}$ makes it to crash and the inclusion of $\mathrm{C}_{9,1}$ speeds up the crash. Also, the tesseral harmonics cause medium periodic (with periods that are less than the moon's orbital period) variations in the periapsis altitude. These variations must be estimated as accurately as possible to devise a maintenance strategy that involves estimation of the magnitude of velocity requirements and frequency of the manoeuvers. Similar trends are observed for other inclinations also. For high life time cases $\left(i=95^{\circ}\right)$, the absence of these harmonics results in low periapsis altitudes during the orbital evolution. This is illustrated in figure 7 . These terms accelerate the periapsis altitude downwards in low life time cases and decrease the rate of change in the periapsis altitude in high life time cases.

\section{Conclusions}

The impact of inclination on the evolution of periapsis altitude is assessed. An inclination of $95^{\circ}$ has the least variation in the periapsis altitude and this variation lies within $\pm 26 \mathrm{~km}$. This choice reduces the maintenance cost considerably. The orbital life time is minimum (16 days) for an inclination of $11^{\circ}$ for low altitude orbits. The specific spherical harmonics that cause this phenomena are $\mathrm{C}_{7,0}, \mathrm{C}_{9,0}$ and $\mathrm{C}_{9,1}$. These terms help to stabilize the periapsis altitude for the $95^{\circ}$ case while being the cause for early crash in the cases of low orbital life times. A proper choice of right ascension of ascending node $\left(0^{\circ}\right.$ for $95^{\circ}$ inclination case $)$ was also found to improve the orbital lifetime and reduce the maintenance cost.

\section{References}

Cook R A 1991 The long term behaviour of near-circular orbits in zonal gravity field; AAS 91-463, AAS/AIAA Astrodynamics Conference. 
Cook R A and Sweetser T 1992 Orbit maintenance for low altitude near circular orbits; AAS 92-185, AAS/AIAA Spaceflight Mechanics Conference.

Konopoliv A S et al 1993 A high resolution lunar gravity field and predicted orbit behaviour; AAS 93-622, AAS/AIAA Astrodynamics Conference.

Konopoliv A S et al 1998 Improved gravity field of the Moon from lunar prospector; Science $\mathbf{2 8 1}$ 1476-1480.
Lemoine F G R et al 1997 A 70th degree lunar gravity model (GLGM2) from Clementine and other tracking data; J. Geophys. Res. 102 No. E7, 16339-16357.

Lozier D et al 1998 Lunar prospector mission design and trajectory support; AAS 98-323, AAS/AIAA Spaceflight Dynamics Conference.

Park Sang-Young and Junkins John L 1995 Orbital mission analysis for a lunar mapping satellite; J. Astro. Sci. 43(2) 207-217. 